Historic, Archive Document

Do not assume content reflects current scientific knowledge, policies, or practices. 



\section{SURPLUS PRICE LIST FOR THE TRADE ONLY SPRING 1927}

Our Small Fruit plant orders are filled direct from the ground-you get your plants fresh and alive. This means much to you.

Prices in this list are for the trade only, and are subject to the stock being unsold when order is received, and prices subject to change without notice.

Certificate of Inspection accompanies each shipment.

While we exercise the greatest care to have our plants true to name, should any stock not prove true to name, we will either refund the purchase money of same, or replace the stock true to name, free; but are not liable for further damage.

Our plants are guaranteed true to name, to be fine, and to prove satisfactory or your money refunded.

Terms: Cash with order, or satisfactory references from unknown parties.

For Fall 1926 and Spring 1927, we quote you special prices on strictly first-class stock, F.O.B. car.

Packing done very carefully for which the charge of material used in packing will be charged you.

Packing will be free of charge if cash accompanies order.

We cannot fill long list of assorted stock calling for only a few of a kind, except at an advance of 20 per cent above printed list.

Prices herein are net-- -no discount.

We can ship under your tags to avoid delay. In shipping season, orders are shipped same day received. 


\section{STRAWBERRY PLANTS}

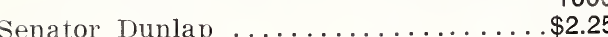

Dr. Burrell ................... 2.25

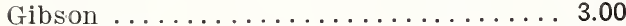

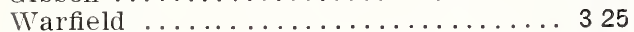

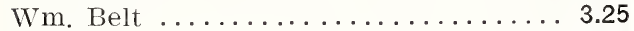

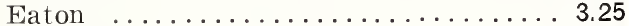

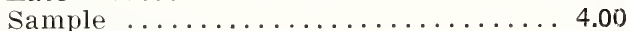

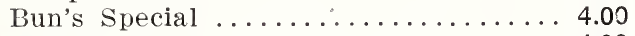

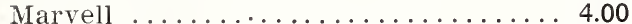

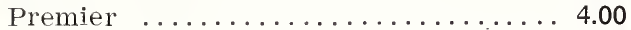

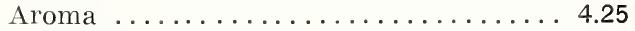

Glenn Mary ....................... 4.25

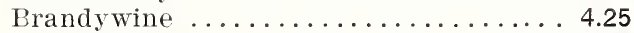

Coopei .......................... 4.50

\section{EVRRBEARING STRAWBERRY PLANTS}

1000

Mastodon $\$ 18.00$

\section{ASPARAGUS ROOTS}

Palmetto and Conover, $1 \mathrm{vr}$. No. $1 \ldots . . \$ 5.00$

Palmetto and Conover, 2 yr. No. $1 \ldots . .7 .00$

Martha Washington, 1 yr. No. $1 \ldots . .6 .00$

Martha Washington, 2 vr. No. $1 \ldots . . .8 .00$

\section{RHUBARB}

Myatts Linneaus and Mammoth,

1 yr. No. 1 ................\$15.00

Myatts Linneaus and Mammoth,

2 yr. No. 1 ................ 25.00

\section{CURRANT PLANTS}

London Market, 1 yr. No. 1 . .\$3.25

London Market, 2 yr. No. $2 \ldots 325$

London Market, 2 yr. No. 1 ... 4.00

London Market, 3 yr. No. 1 . . 4.50

Wilder, 1 yr. No. 1 ........ 3.50

Wilder, 2 yr. No. 1 ........ 4.50

Cherry, Fay's Prolific and Red

Cross, 2 yr. No. $2 \ldots \ldots \ldots .5 .00$

1000

$\$ 30.00$

30.00

35.00

40.00

30.00

40.00

40.00

\section{GOOSEBERRY PLANTS}

1001000

Downing, 2 yr. No. $1 \ldots \ldots \ldots . . \$ 7.00 \$ 60.00$

\section{BLACKBERRY PLANTS} No. 1 Suckers

Eldorado ................ \$1.25

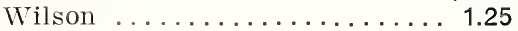

Blask Diamond ............ 1.60

Blowers ................. 1.40

Miersereau ............... 1.40
Blackberry Root Cuttings

$100 \quad 1000$

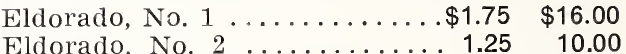

Blowers, No. $1 \ldots \ldots \ldots . \ldots 2.00 \quad 17.00$

Mersereau, No. 1 ......... 2.0017 .00

Mersereau, No. $2 \ldots \ldots \ldots \ldots \ldots 1.2511 .00$

\section{Raspberry No. 1 Suckers} $100 \quad 1000$

King ................\$1.25 \$10.00

Cuthbert .............. $1.40 \quad 12.00$

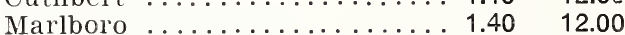

Victory ................. $1.75 \quad 15.00$

\section{Strong 2 Yr. Transplants}

1001000

King ..............\$2.00

St. Regis ................ 225

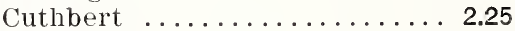

Marlboro .............. 225

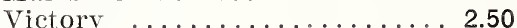

$\$ 18.0 \mathrm{C}$

Black Raspberry Tips No. 1 $100 \quad 1000$

Cumberland ............\$1.40 \$12.00

Kansas ................ $1.40 \quad 12.00$

Gregg .................... $1.40 \quad 12.00$

Plum Farmer ............. $1.40 \quad 12.00$

Royal Purple tips .......... 2.2J 20.00

\section{TRANSPLANTS}

$100 \quad 1000$

Royal Purple ............\$3.00 \$27.00

Plum Farmer............. 2.25

Kansas ............... 2.25

Cumberland ............ 2.25

\section{Currant Cuttings 7 to 9 inches}

1000

L ondon Market ...............\$3.00

Cherry and Fay's Prolific ........4 4.00

Red Cross .................... 4.00

\section{Grape Cuttings 8 to 12 inches}

Concord .................\$1.60

Ives and Champion ........... 2.00

Moore's Early .................... 2.25

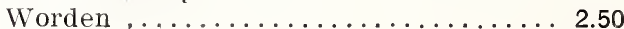

Niagara . . . . . . . . . . . . . 2.75

Delaware ................... 2.75

Campbell's Early .............. 4.00

\section{GRAPE VINES-1 Yr. Old No. 2}

Concord and Champion .........\$14.00 Niagara, Moore's Early, Worden ..... 20.00

Agawam, Delaware ............ 25.00

Brighton, Salem ............ 25.00 
GRAPE VINES-Red Varieties $100 \quad 1000$

Agawam, 1 yr. No. $1 \ldots \ldots \ldots . \$ 5.00 \$ 40.00$

Agawam, 2 yr. No. 1 ....... 6.00

Catawba, 1 yr. No. $1 \ldots \ldots . . .5 .00$

Catawba, 2 yr. No. 1 ...... 6.00

Brighton, 1 yr. No. 1 . . . . 5.00

Brighton, 2 yr. No. $1 \ldots \ldots \ldots 7.00$

Moore's Diamond, 1 yr. No. 1 . 5.00

Delaware, 1 yr. No. 1 ...... 5.00

Niagara, 1 yr. No. $1 \ldots \ldots \ldots . . .3 .50$

Niagara, 2 yr. No. $1 \ldots \ldots \ldots . . .5 .00$

Salem, 1 yr. Nó. $1 \ldots \ldots \ldots . . \ldots 0$

\section{GRAPE VINES-Black Varieties}

$100 \quad 1000$

Moore's Early, 1 yr. No. $1 \ldots . \$ 4.00 \$ 35.00$

Moore's Early, 2 yr. No. 1 ... $5.00 \quad 45.00$

Worden, 1 yr. No. 1 ...... $4.50 \quad 40.00$

Worden, 2 yr. No. $1 \ldots \ldots . .5 .00 \quad 45.00$

Champion, 1 yr. No. $1 \ldots \ldots .2 .50 \quad 20.00$

Champion, 2 yr. No. $1 \ldots \ldots .3 .50 \quad 30.00$

Concord, 1 yr. No. $1 \ldots \ldots . .2 .00 \quad 18.00$

Concord, 2 yr. No. $1 \ldots \ldots .44 .00 \quad 35.00$

Concord, 3 yr. No. 1 ...... $4.50 \quad 40.00$

Your correspondence solicited,

L. J. RAMBO \& SONS

R.F.D. 1, Bridgman, Michigan 
